\title{
Immediately Loaded Zygomatic Implant Retained Maxillary Obturator used in the Management of a Patient following Total Maxillectomy
}

\author{
Chris J Butterworth
}

\begin{abstract}
Aim: This study aimed to report on the use of specialized zygomatic oncology osseointegrated implants to assist in the management of a patient struggling to cope with a large maxillary obturator prosthesis following a low-level total maxillectomy.
\end{abstract}

Background: Maxillary obturator prostheses are commonly used in the rehabilitation of patients following maxillary resection. Whilst these prostheses can be retained adequately with remaining maxillary teeth and/or implants, as the horizontal component of the resection increases, the retention, and support of the obturator are increasingly difficult to manage.

Case description: A 72-year-old female patient was referred with problems tolerating a large removable maxillary obturator prosthesis, following total maxillectomy three years previously. Despite successful disease control without the need for adjuvant therapy, the patient functioned poorly with the obturator prosthesis which was poorly retained and supported by the large defect. In addition, the movement of the obturator resulted in soreness and ulceration, and she relied on the heavy use of denture fixative to assist in the wearing of the obturator prosthesis. Following a radiographic investigation, four zygomatic oncology osseointegrated implants were placed into the residual zygomatic bodies and within a week were used to provide much-improved support and retention for the large obturator prosthesis, resulting in successful amelioration of the patient's pre-existing difficulties.

Conclusion: The use of specialized remotely-anchored osseointegrated zygomatic implants can provide a means of effective support and retention for large prosthetic obturators even where the horizontal component of the maxillary resection is large. The density of the zygomatic bone provides excellent initial stability for these implants which can be used immediately to support

Clinical significance: The use of zygomatic oncology implants provides an improved means of retaining and supporting maxillary obturator prostheses where these are required following partial or total maxillary resection.

\section{Consultant}

Department of Oral and Maxillofacial Surgery, Aintree University Hospital NHS Foundation Trust, Lower Lane, Liverpool, UK

Corresponding Author: Chris J Butterworth, Consultant, Department of Oral and Maxillofacial Surgery, Aintree University Hospital NHS Foundation Trust, Lower Lane, Liverpool, UK, Phone: 0151706 5205; e-mail: c.butterworth@ liv.ac.uk
Keywords: Maxillary obturator prosthesis, Maxillectomy, Oral cancer, Osseointegrated implants, Zygomatic oncology implants, Zygomatic implants.

How to cite this article: Butterworth CJ. Immediately Loaded Zygomatic Implant Retained Maxillary Obturator used in the Management of a Patient following Total Maxillectomy. Int J Head Neck Surg 2018;9(2):94-100.

Source of support: Nil

Conflict of interest: None

\section{BACKGROUND}

Prosthetic obturation of maxillary defects is a wellestablished treatment modality which is used extensively around the world. Whilst it provides challenges for patients and clinicians alike, it is a much simpler approach than the use of microvascular surgical free flap reconstruction and can provide similar quality of life and functional outcomes for patients especially in low-level maxillary defects. ${ }^{1}$ The size of the maxillary defect in the horizontal plane together with the quality and number of the remaining maxillary teeth as also important predictors of prosthetic success. ${ }^{2}$ In the edentulous patient or whether a large horizontal part of the maxilla has been removed, the use of dental implants is increasingly indicated and can provide much-needed stability and retention for the obturator prosthesis in these cases. ${ }^{3}$ Zygomatic implants were originally designed for use in these patients as they can provide in-defect support and retention as they are anchored remotely in the zygomatic bone which is preserved in a low-level maxillary resection. ${ }^{4}$ These implants can be placed at the time of primary resective surgery ${ }^{5}$ or at a subsequent timepoint to help with the retention and stability of an obturator prosthesis. ${ }^{6}$ As confidence in this treatment approach has increased, an early prosthetic loading approach has been undertaken by some clinicians, rather than leaving the implants to formally osseointegrate for a number of months prior to using them.

This article describes the early use of specialized zygomatic osseointegrated implants (zygomatic oncology implants) in the management of a patient who had previously undergone a sub-total maxillectomy $\left(\right.$ Brown $^{3}$ $2 \mathrm{~d}$ resection) and was struggling with her large obturator prosthesis. 


\section{CASE DESCRIPTION}

A72-year-old Caucasian female patient was referred to the Merseyside Regional Head and Neck Cancer Centre with problems functioning with her large maxillary obturator prosthesis (Fig. 1). The main presenting issues were a lack of retention and stability, discomfort during chewing, blockage of the nasal airway and a lack of confidence when eating in public. The patient admitted to using significant amounts of denture fixative to help retain the prosthesis and to make it more comfortable; however, the effectiveness of this solution was short-lived and largely unsatisfactory. Over the previous three years, the obturator prosthesis had been remade unsuccessfully on four occasions to try and improve the patient's comfort function. The patient was otherwise well with no significant systemic pathology and was a non-smoker. She had been diagnosed three years previously with a desmoplastic fibroma (DF) in the maxilla which had been resected via a sub-total maxillectomy (Brown ${ }^{3}$ Class 2d) in January 2014 with the retention of a small amount of hard palate and the maxillary tuberosities

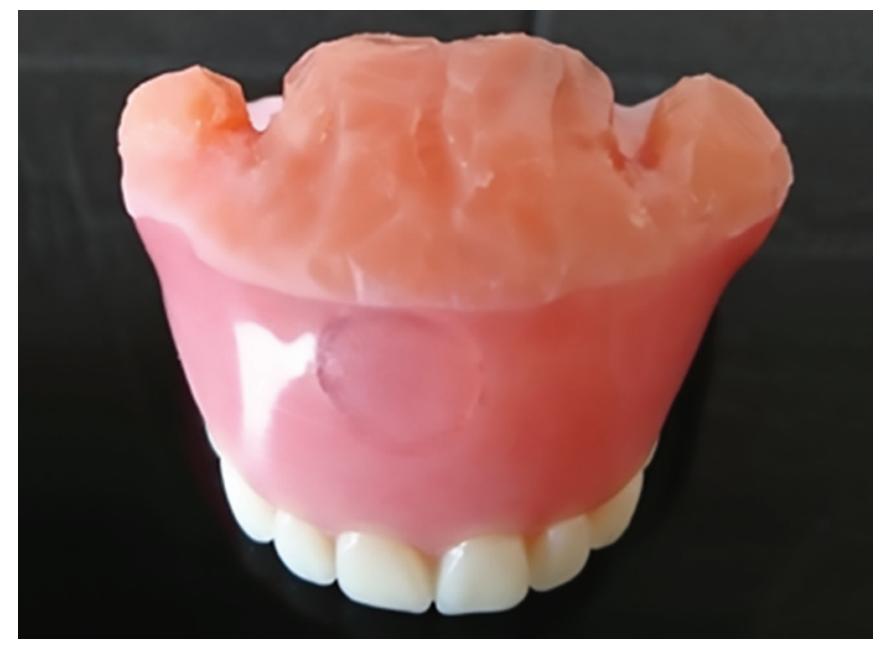

Fig. 1: Large maxillary obturator prosthesis with soft resilient lining to engage the residual nasal and antral structure

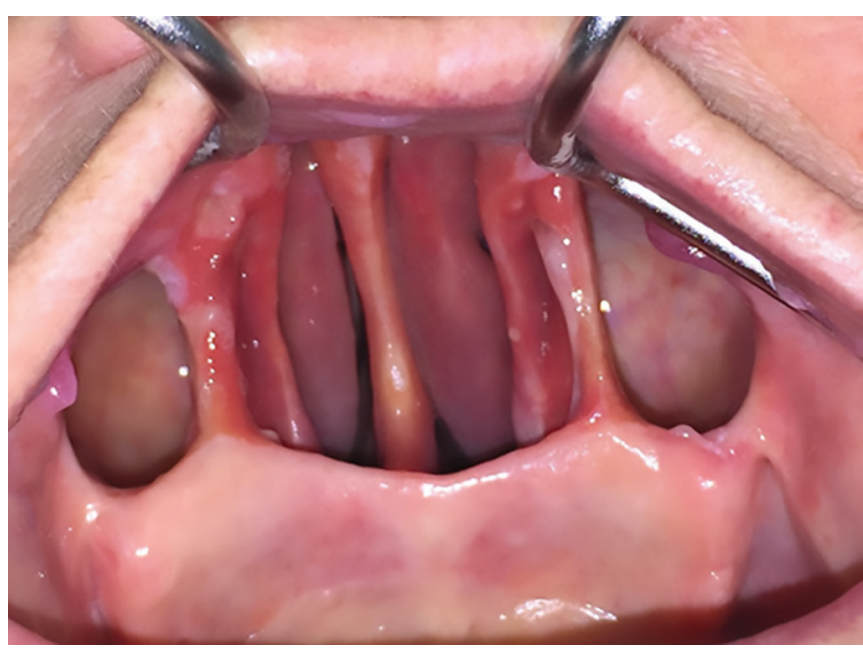

Fig. 2: Pretreatment clinical situation following previous sub-total maxillectomy. Note the evidence of residual denture fixative used to retain the conventional obturator prosthesis bilaterally. No defect skin grafting had been undertaken at the primary operation, and as a result, the defect was extremely sensitive and prevented the engagement of any significant undercut to retain the prosthesis (Fig. 2). She was partially dentate and wore a precision retained the mandibular partial denture. Remnants of denture fixative were evident, but otherwise, the maxillectomy defect was healthy with no evidence of local tumor recurrence. A panoramic radiograph (Fig. 3) confirmed the significant maxillary resection and the lack of alveolar bone remaining to place standard endosseous dental implants. A subsequent CT scan of the patient demonstrated the extent of the previous resection but confirmed the excellent bone volume bilaterally in the patient's zygomatic bodies for the placement of multiple zygomatic oncology implants in a remote anchorage protocol (Fig. 4).

Under day case general anesthesia, access was obtained to both zygomatic bodies via an intra-oral approach with subperiosteal dissection over the external part of zygomatic prominence and sub-mucosal dissection on the medial aspect. Two zygomatic oncology implants $(37.5 \mathrm{~mm}$; Southern Implants Ltd, Irene; South Africa) were placed in a horizontal fashion on each side with high initial stability, taking care not to extrude the tips of the implants too far through the zygoma and ensuring prosthetic heads of the implants were in a useful and restorable position (Fig. 5). The intra-oral incisions were closed with resorbable sutures, and Implant abutments were placed and torqued into position on all four implants. Impression copings were attached to the abutments and splinted together with light-cured acrylic tray material (Individo ${ }^{\circledR}$ Lux, VOCO Gmbh, Germany). The maxillary defect undercuts were then blocked out using surgical gauze prior to taking a maxillary impression (Fig. 6). The patient's initial obturator was cut back to allow it to be seated over the implant heads and it was relined with a soft-lining material (Ufigel SC, VOCO Gmbh; Germany). A subsequent radiograph demonstrated good positioning of the implants within the zygomatic bodies (Fig. 7).

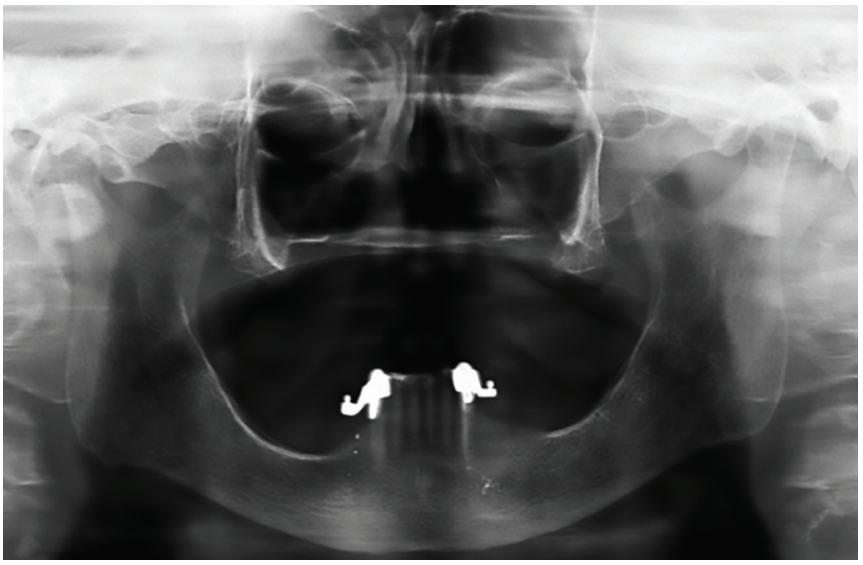

Fig. 3: Pretreatment panoramic radiograph demonstrating severe maxillary bone defect 


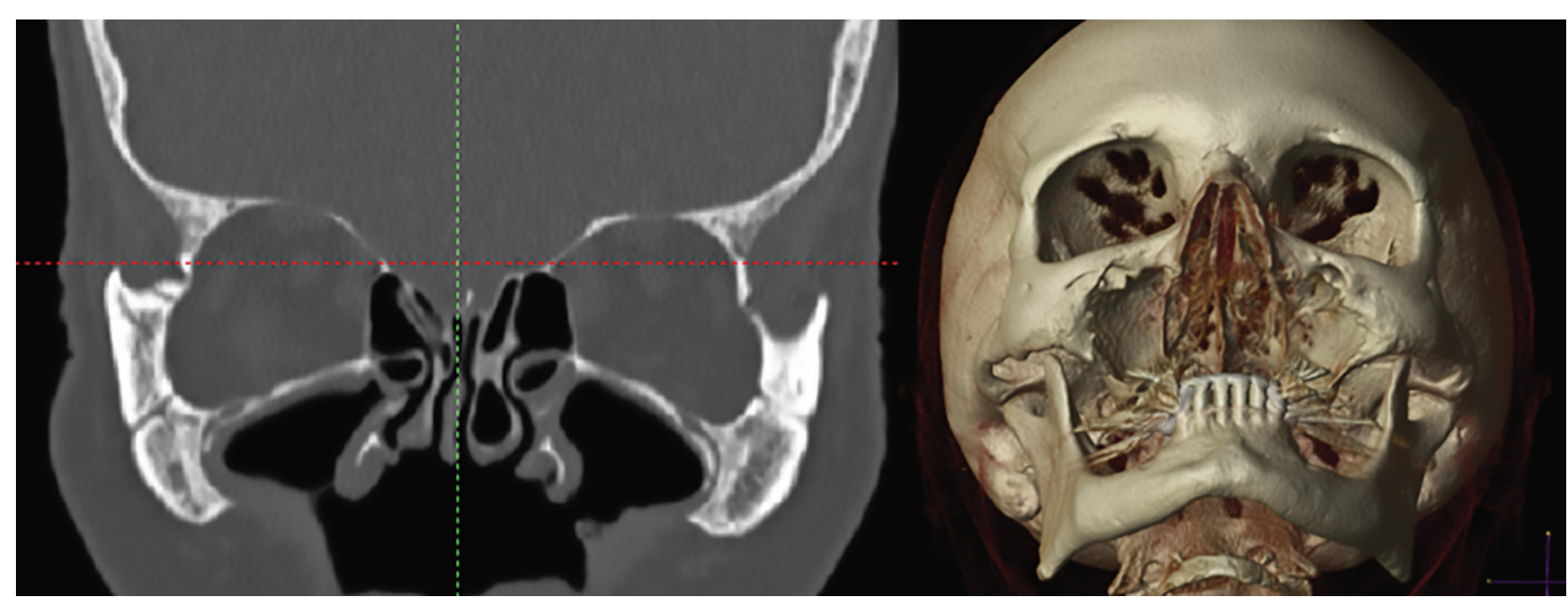

Fig. 4: Coronal CT scan image and 3D reconstruction showing the extent of the maxillary resection and the presence of adequate bone within the zygomatic bodies bilaterally to support the placement of zygomatic implants

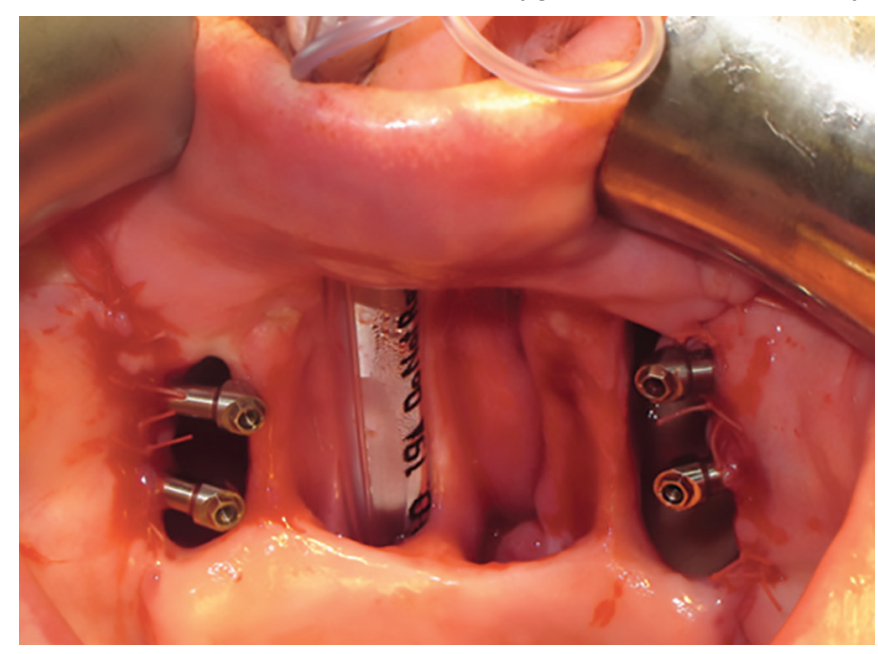

Fig. 5: Surgical view of maxillary defect following placement of four zygomatic oncology implants via an intra-oral approach. Note the polished surfaces of the implants extending into the oral cavity to facilitate cleaning and soft tissue health

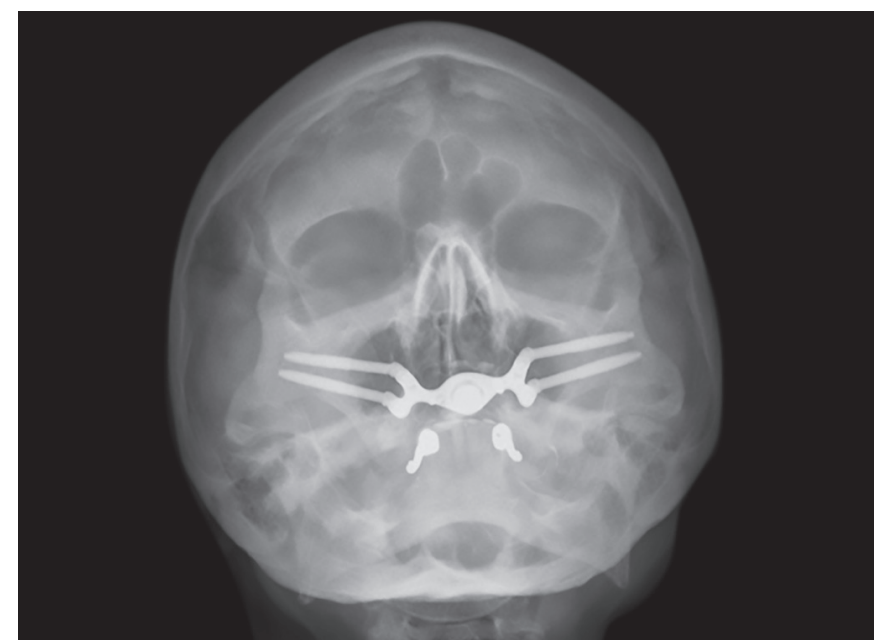

Fig. 7: Post-operative facial plain radiograph demonstrating the position of the zygomatic implants within the bodies of the zygomatic bones and the connecting metal bar in situ

The impressions were subsequently poured by the prosthetic technician who then constructed a cobaltchrome based bar construction which incorporated a large

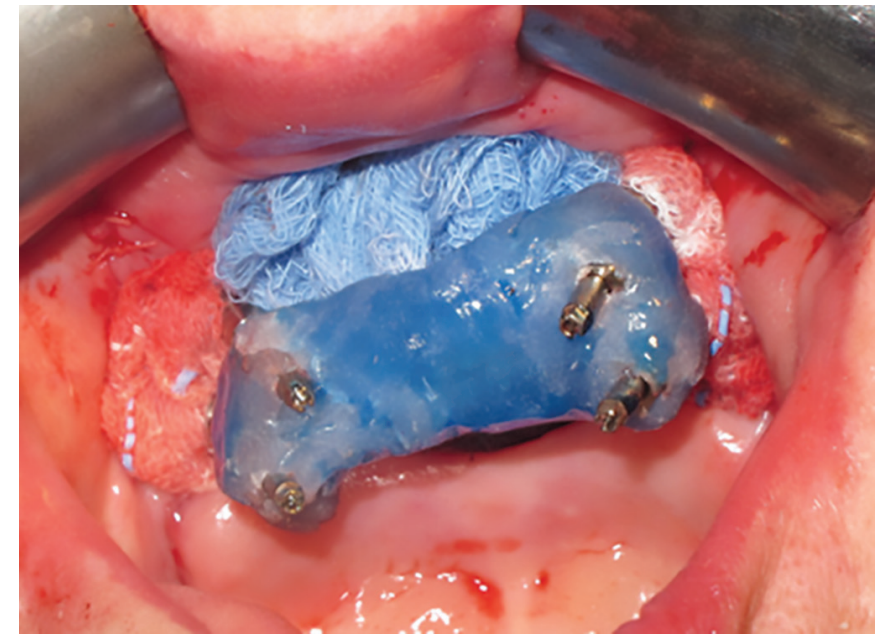

Fig. 6: Splinting of the implant abutment impression copings at the time of surgery with light-cured acrylic material in preparation for the master impression together with packing of the defect undercuts with gauze

central magnet (Technovent; Bridgend; Wales) cured into a central housing (Fig. 8). In addition, an acrylic pick up housing was constructed (Fig. 9) to allow conversion of the patient's existing obturator prosthesis. One week following implant insertion, the relined obturator was removed and the new zygomatic implant retained bar construction was fitted (Fig. 10). The acrylic pick up housing was then seated over the bar, and the patient's existing obturator was modified significantly to remove the soft superior components which had previously engaged the sensitive undercuts within the nasal and sinus cavities. Once the prosthesis could be seated into the correct position, the acrylic housing was bonded to the overlying prosthesis using chairside cold-cure acrylic material (Tokuyama Rebase II; Tokuyama dental corporation; Japan) taking care not to extrude unwanted material beneath the bar or around the shafts of the implants (Fig. 11). The occlusion was checked and the patient instructed in the removal and cleaning of the prosthesis and implant bar apparatus (Fig. 12). 
On review, the patient reported a significant improvement in her previous symptoms and was now able to breathe through her nose and function with her obturator to a much higher level. All intra-nasal discomfort was gone and her chewing was much improved with no

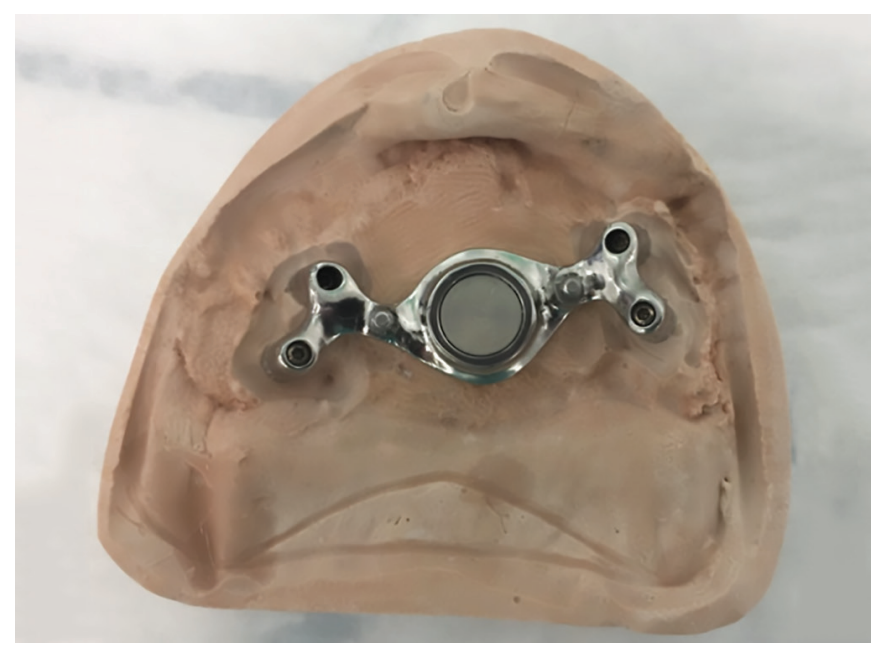

Fig. 8: Cobalt-chrome cast bar containing a large central magnet which is located in a central housing and luted in place with acrylic

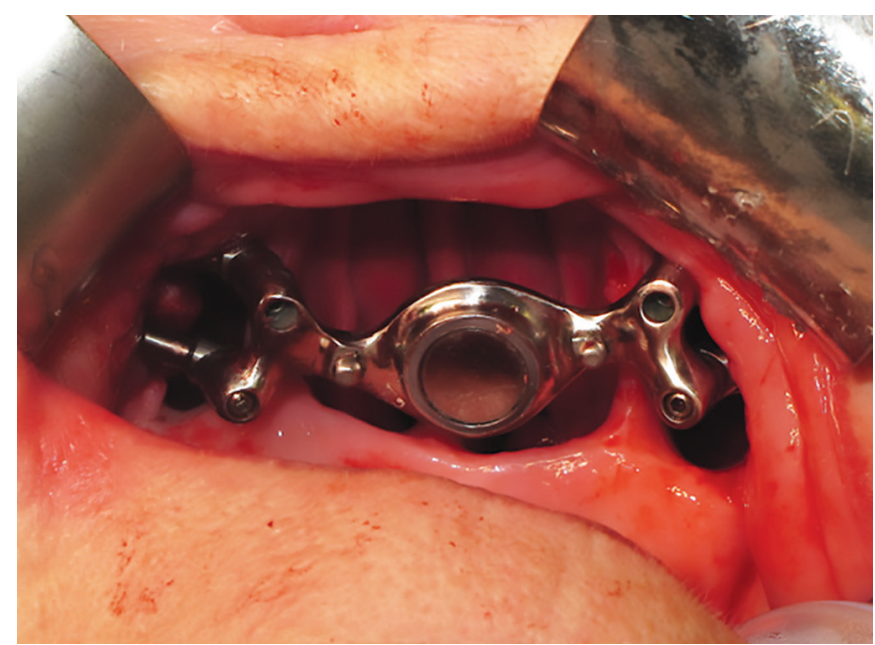

Fig. 9: Acrylic pick-up device which is fitted directly over the implantretained bar and retained by a corresponding magnet. This device is used to convert the patient's existing obturator into a precision retained and supported prosthesis once the bar is fitted
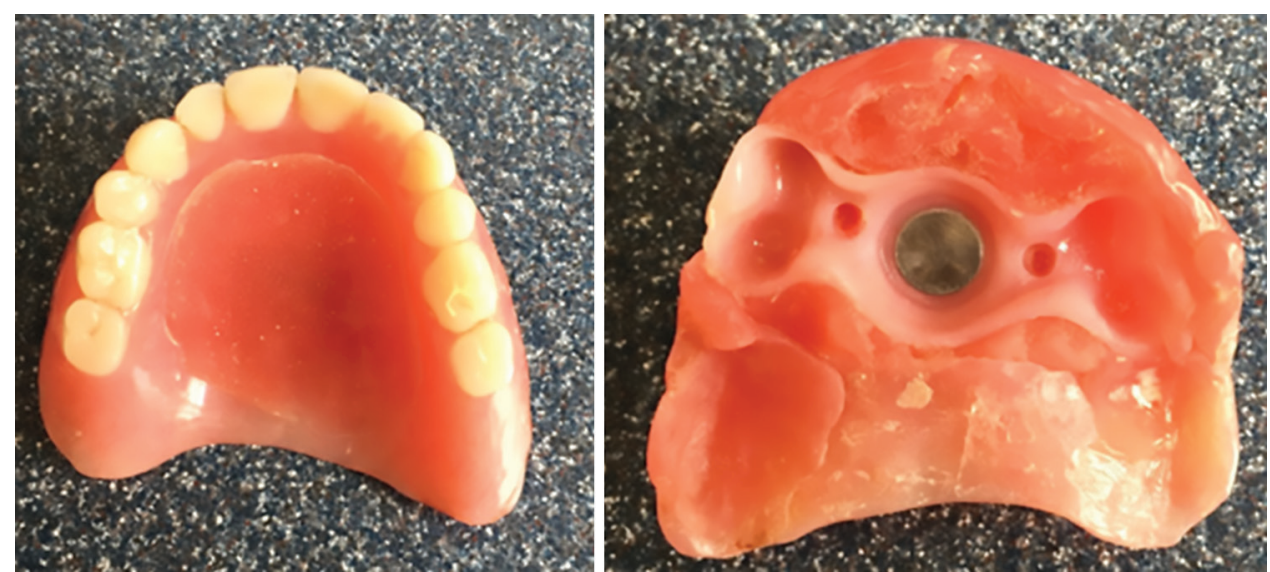

Fig. 11: The patient's original obturator prosthesis following removal of the soft bung material and luting of the acrylic pick up a device significant obturator mobility. She no longer required to use denture fixative and report overall a much-improved quality of life from the procedure. She experienced minor episodes of oro-nasal fluid leakage during the initial healing period which was managed with small additional modifications to the prosthesis. A new obturator prosthesis (Fig. 13) was subsequently constructed for the patient after four months to ensure the best fit and oronasal seal was achieved. The patient has been followed closely for 9 months since implant placement with no evidence of peri-implant problems or infection, mainly due to the polished surface of the zygomatic oncology implants which facilitate cleaning.

\section{DISCUSSION}

The use of maxillary obturator prostheses for the management of patients following maxillectomy procedures has a long and well-reported history and in many parts of the world is the default treatment option. On the face of it, these prostheses can provide solutions to the problems caused by maxillectomy, namely closure of the oro-nasal defect, facial and lip support, dental rehabilitation as well as providing restoration of

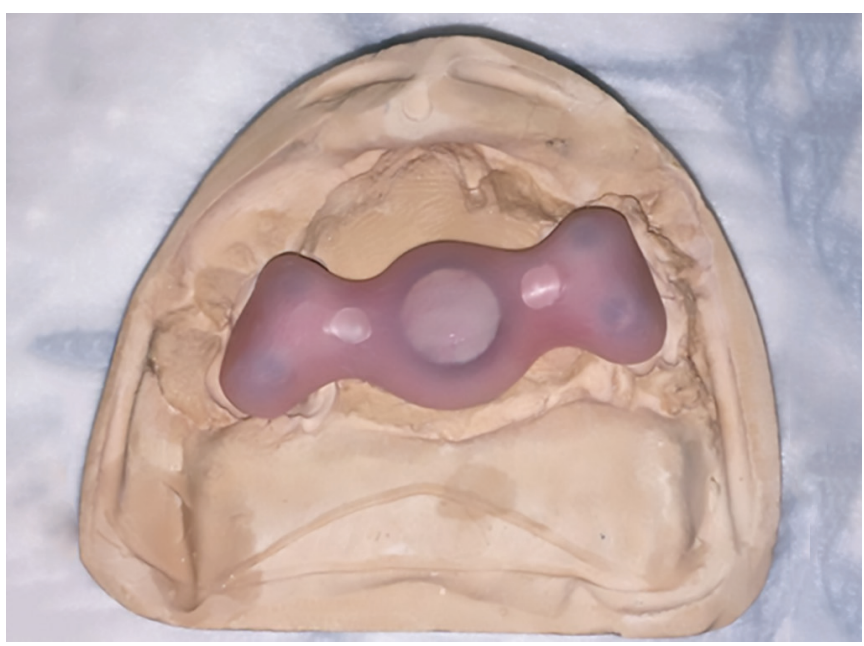

Fig. 10: Screw-retained implant bar fitted one-week post-surgery providing in-defect support and retention for the prosthesis 


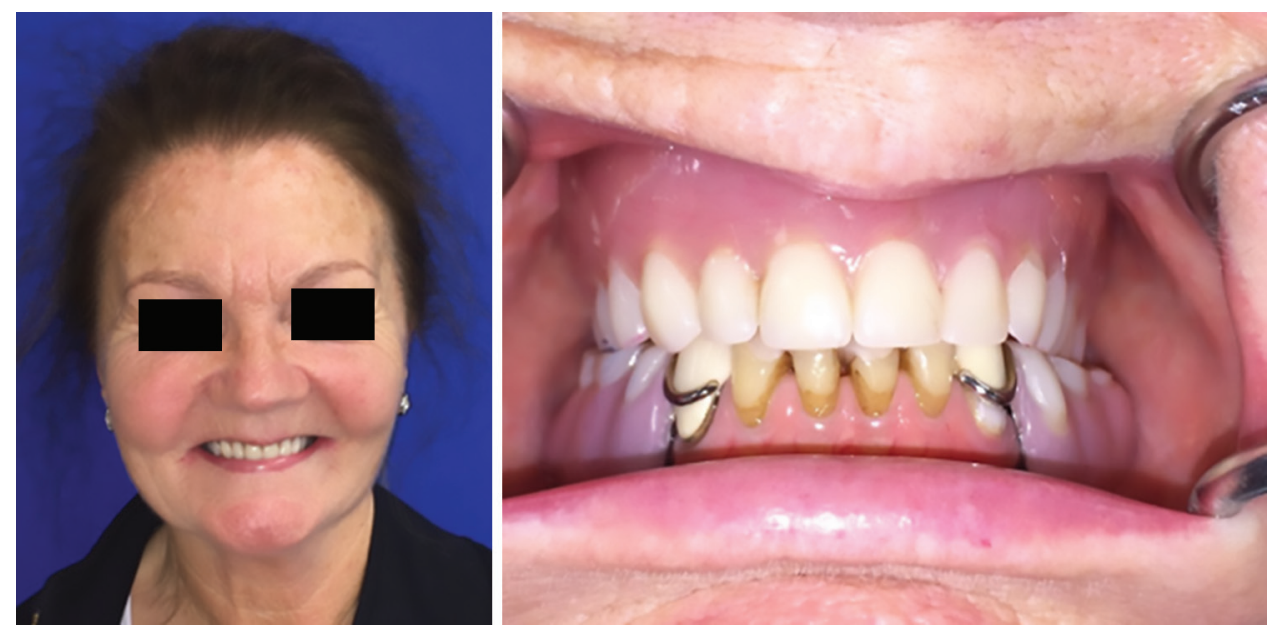

Fig. 12: Modified original obturator in-situ 6 weeks post surgery
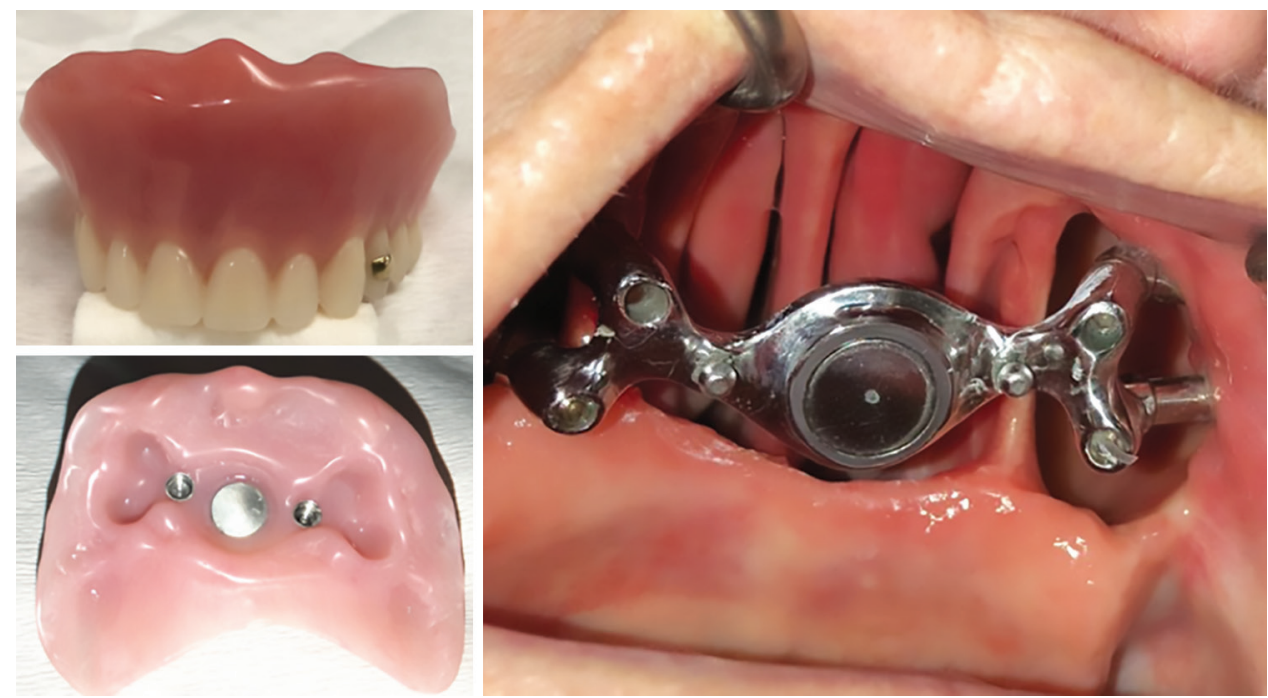

Fig. 13: New obturator prosthesis and clinical situation 9 months following implant placement. Note the excellent peri-implant soft tissue health and the absence of any mucosal trauma

speech and swallowing. Obturator prostheses can also be provided immediately at the time of the resective surgery removing the need for the patient to adapt to a period without their dentition and the cosmetic and psychological consequences which this brings for some patients. The use of maxillary obturators is best reserved for low-level maxillary defects where the orbital floor, zygomatic prominence, and some para-nasal support is retained. This would correspond to a level 1 or 2 resection using the Brown ${ }^{3}$ classification. For these low-level resections, obturator prostheses can work well depending on the horizontal component of the resection and the remaining maxillary dental status of the patient. Where the resection is unilateral, and the canine on the ips-lateral side remains, then the use of an obturator which gains support and retention from the remaining teeth can work extremely well. Okay, et $\mathrm{al}^{2}$ have produced useful guidelines on the design of maxillary obturators depending on the size of resection and the remaining teeth present. In addition to the appropriate framework design, surgical modifications such as alveolar resection along the axis of a dental socket, removal of the inferior turbinate, application of a split-skin graft to the cheek defect and ensuring wrapping of the medial bone margin with palatal mucosa are all important factors in maximizing the success and tolerance of the obturator prosthesis by the patient.

In the edentulous maxillectomy patient, the residual alveolar ridge provides very little retention for the prosthesis, and the defect has to be engaged with the bung of the prosthesis to gain retention. Many patients find this uncomfortable, and as a result, the amount of undercut engaged is often limited, and this reduces the overall retention and tolerance of the prosthesis. As the horizontal component of the resection increases and especially in cases of sub-total maxillectomy, there is no real support for the prosthesis whatsoever, and patients are often reliant on denture fixatives with prostheses which constantly traumatize the sensitive structures of the nose. Moreno ${ }^{1}$ confirmed this from their study that where the horizontal maxillectomy component was large, 
patients reported better quality of life and functional outcomes (speaking/chewing) when restored with a free flap compared to a conventional (non-implant-retained) obturator prosthesis.

The use of zygomatic implants has revolutionized the prosthodontic rehabilitation of the low-level total maxillectomy patient and provided a much simpler but effective solution which allows patients to function well with an obturator prosthesis which is well retained and supported both in and out of function. ${ }^{7}$ This is especially helpful in the elderly patient who may not be fit for or wish to undergo complex free flap surgery with all its potential complications and drawbacks. The ability to engage the highly cortical bones of the zygomatic bodies provides a high strength remote connection to the facial skeleton which can be utilized to provide support and retention for the prosthesis exactly where it is required-the center of the defect. In the case presented, four implants were successfully placed due to the abundant bone available in the patient's zygomas, and this supported a highly rigid bar-splinted apparatus containing a large magnet. The implant-supported bar provided directed support for the prosthesis during mastication without the need to engage and traumatize the inner nasal and antral structures. The magnet provided an excellent means for the patient to locate and fit the prosthesis in place over the bar as once the prosthesis is introduced into the mouth, it is guided into place by the magnetic forces between the bar-magnet and the opposing magnet on the intaglio surface of the prosthesis. Compared to other precision attachments, the author's experience is that most patients find the use of such a magnetic retention system more favorable and easier to manage. The use of implants also reduces the vertical height of the obturator which facilitates its an insertion by the patient, especially if the patient has a degree of trismus following treatment. As the implant bar-apparatus are now providing the retention and support, the prosthesis can be made entirely of highly polished acrylic materials which are extremely durable and easy to clean rather than softer more degradable and infiltrative materials designed to cushion the fitting surface of the prosthesis.

This case also demonstrates the effective use of specifically modified zygomatic implants (Oncology Zygomatic implant; Southern Implants Ltd; Irene; South Africa) (Fig. 14) which has been modified in its coronal section to be highly polished, specifically for use in maxillectomy situations where this part of the implant extends into the maxillectomy cavity. This facilitates the effective cleaning of the implant by the patient and prevents the accumulation of plaque and debris at the

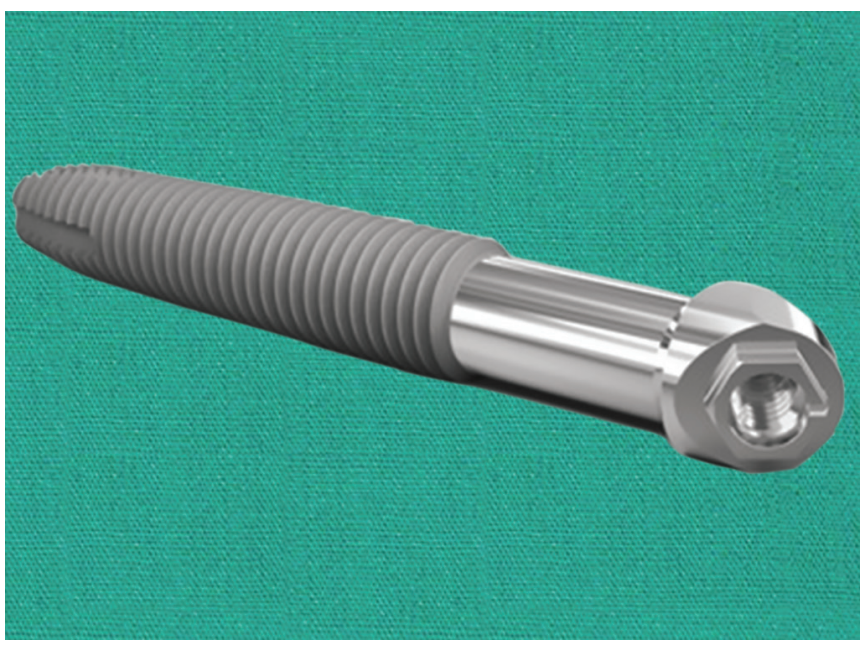

Fig. 14: Zygomatic oncology implant; note the polished portion of the implant designed to allow use in maxillectomy defects and the 55 degrees angled head design

soft tissue interface, thereby reducing peri-implant inflammation.

The practice of immediate loading of zygomatic implants is increasingly supported as a viable and effective treatment modality. ${ }^{8}$ In the maxillectomy situation, a few authors have utilized this approach with the attendant benefit to patients and without experiencing zygomatic implant failure. ${ }^{5,9}$ The benefits to patients with this approach is self-evident, namely a more rapid rehabilitation without a prolonged period of temporization with a clumsy, uncomfortable and compromise prosthesis. The highly cortical nature of the zygomatic bones facilitate insertion of implants with high primary stability as long as the volume of bone is adequate and the surgical technique well executed. Splinting of contralateral implants, ideally in a quad configuration, provides a highly stable platform to support and retain a large prosthesis as has been demonstrated by this case. The use of the custommade acrylic transfer jig (Fig. 9) allows the patient's existing prosthesis to be modified at a very early stage following surgery and this, in turn, reduces the adaptation period for the patient as they can continue to wear the prosthesis to which they have already adapted to form a cosmetic and speech perspective. The patient reported much-improved quality of life following the procedure and certainly further ongoing research into the effectiveness of these procedures compared to other more complex reconstructive techniques is required.

\section{CONCLUSION}

The use of remotely anchored zygomatic oncology implants can provide a stable and retentive platform to support the retention and support for maxillary obturator prostheses even following total maxillectomy. 


\section{CLINICAL SIGNIFICANCE}

Zygomatic implants provide an additional tool for clinicians in the prosthetic management of maxillary and mid-facial tumors. The high primary stability achieved within the zygomatic bones allows immediate or early restoration within a matter of weeks and provides invaluable stability and anchorage for specialized obturator prostheses with much-improved function compared to standard tissue-borne prostheses.

\section{REFERENCES}

1. Moreno MA, Skoracki RJ, Hanna EY, Hanasono MM. Microvascular free flap reconstruction versus palatal obturation for maxillectomy defects. Head Neck. 2010;32(7):860-868.

2. Okay DJ, Genden E, Buchbinder D, Urken M. Prosthodontic guidelines for surgical reconstruction of the maxilla: a classification system of defects. J Prosthet Dent. 2001;86(4):352-363.

3. Brown JS, Shaw RJ. Reconstruction of the maxilla and midface: introducing a new classification. Lancet Oncol. 2010; 11(10):1001-1008.
4. Parel SM, Brånemark PI, Ohrnell LO, Svensson B. Remote implant anchorage for the rehabilitation of maxillary defects. J Prosthet Dent. 2001;86(4):377-381.

5. Boyes-Varley JG, Howes DG, Davidge-Pitts KD, Brånemark I, McAlpine JA. A protocol for maxillary reconstruction following oncology resection using zygomatic implants. Int J Prosthodont. 2007;20(5):521-531.

6. Schmidt BL, Pogrel MA, Young CW, Sharma A. Reconstruction of extensive maxillary defects using zygomaticus implants. J Oral Maxillofac Surg. 2004;62(9 Suppl 2): 82-89.

7. Dattani A, Richardson D, Butterworth CJ. A novel report on the use of an oncology zygomatic implant-retained maxillary obturator in a paediatric patient. Int J Implant Dent. 2017;3(1):9.

8. Chrcanovic BR, Albrektsson T, Wennerberg A. Survival and Complications of Zygomatic Implants: An Updated Systematic Review. J Oral Maxillofac Surg. 2016;74(10):1949-1964.

9. Butterworth CJ, Rogers SN. The zygomatic implant perforated (ZIP) flap: a new technique for combined surgical reconstruction and rapid fixed dental rehabilitation following low-level maxillectomy. Int J Implant Dent. 2017;3(1):37. 